\title{
THE EFFECT OF ALUMINUM CONTENT ON THE MICROSTRUCTURE AND CAVITATION WEAR OF FEAI INTERMETALLIC ALLOYS
}

\author{
Robert JASIONOWSKI*, Dariusz ZASADA**, Wojciech POLKOWSKI* \\ *Institute of Basic Technical Sciences, Maritime University of Szczecin, 70-205 Szczecin, ul. Podgórna 51-53, Poland \\ ${ }^{* *}$ Faculty of Advanced Technology and Chemistry, Military University of Technology, 00-908 Warszawa, ul. Kaliskiego 2, Poland \\ r.jasionowski@am.szczecin.pl, dzasada@wat.edu.pl,wpolkowski@wat.edu.pl
}

\begin{abstract}
Intermetallic-based alloys (so called intermetallics) of the Fe-Al binary system are modern construction materials, which in recent decades have found application in many branches of the power, chemical and automotive industries. High resistance of FeAl based alloys to cavitational erosion results first of all from their high hardness in the as-cast state, large compressive stresses in the material, as well as homogeneous structure. In the present paper, the effect of aluminum content on the microstructure, texture and strain implemented upon cavitation wear of FeAl intermetallic alloys, have been analyzed by field emission gun scanning electron microscopy (FEG SEM) and electron backscatter diffraction (EBSD) analysis. Obtained results of structural characterization indicates that with increasing aluminium content effects of orientation randomization (weakening of $\langle 100>/ / N D$ casting texture), grain refinement and rising of mechanical strenght (and so cavitational resistance) take place.
\end{abstract}

Key words: Intermetallic Alloys, Cavitation, Cavitation Wear, Electron Backscattered Diffraction (EBSD)

\section{INTRODUCTION}

The use of alloys based on intermetallic phases, so called aluminides, in near future can prove to be a far-reaching solution. This term describes materials based on intermetallic phases from $\mathrm{Ni}-\mathrm{Al}$, Ti-Al and Fe-Al, which are characterized by: high mechanical strength, high elastic constant, low density when compared to steel, good high-temperature creep resistance, and high resistance to oxidation and corrosion in aggressive environment (Bojar and Przetakiewicz, 2006; Bystrzycki et al., 1996).

From among intermetallic alloys of the Fe-Al system, those based on the FeAl intermetallic phase of the B2-type structure deserve particular attention due to their unique technological properties. This phase is being found in a wide range of aluminum content $(36 \div 51 \%$ at.), which directly affects the mechanical properties of these alloys. The increase of aluminum content is reflected in their density, oxidation resistance, hardness and the course of crystallisation. Intermetallic alloys of the Fe-Al system are being obtained by classical methods of melting in crucible induction furnaces. The most frequently used methods are: air induction melting and vacuum induction melting. The production of intermetallics by these methods allows obtaining the materials of high purity, homogeneous and coarse-grained structure with minimum porosity and without internal and surface cracks. The FeAl intermetallic-based alloys are characterized by low density, high mechanical properties, large high-temperature resistance as well as corrosion and abrasive wear resistance. These alloys show also several times larger resistance to cavitational erosion, slightly increased with aluminum fraction, when compared to the $\mathrm{Fe}, \mathrm{Cu}$ and $\mathrm{Al}$ alloys examined on a streaming-blowing test bed.

Higher resistance of FeAl based alloys to cavitational erosion results first of all from their high hardness in the as-cast state, large compressive stresses in the material, as well as homogeneous structure. The mechanism of cavitational destruction of FeAl intermetallic alloys is initiated at the triple points of grain boundary contact, i.e. within the area where first decrements appear. In the incubation period, a plastic strain of the surface layer occurs as well as the phenomenon of grain uprising and collapse (Jasionowski et al., 2010, 2011). In these work a surface changes during cavitational wear were analyzed mainly by light and electron microscopy. In the present paper these experimental data are supplemented with results of electron backscatter diffraction (EBSD) analysis. It has been previously shown (Scgwartz et al., 2009), that this method is an efficient tool of characterization of local strain distribution in surface heavily damaged after machining. In the recent years, the EBSD-based analysis concerns the understanding of local damage in polycrystalline materials induced by several types of mechanical degradation, however mainly dealing with a fatigue damage (e.g. El Bartali et al., 2008; Blochwitz et al., 1996). As so far only a little attention has been paid in this field to the cavitation analysis (e.g. Stella et al., 2014)

The aim of present paper is determination of the effect of aluminum content on the microstructure and cavitation wear of FeAl intermetallic alloys.

\section{INVESTIGATED MATERIALS}

Detailed examinations of the resistance to cavitational wear was carried out on five FeAl intermetallic-based alloys (in the ascast state) with aluminum fraction from 36 to $48 \%$ at. and monophase structure of the ordered solid solution of aluminum in iron lattice (B2-type structure), containing the following micro-additions: molybdenum, zirconium, boron and carbon. The alloys were produced by stage melting in a Leybold-Heraeus vacuum induction furnace of the IS-5/III type, at a temperature of $1500 \div 15500 \mathrm{C}$ and in a vacuum of approximately 0.001 Torr.

The chemical composition of the examined materials and the selected mechanical properties are presented in Tab. 1. 
Robert Jasionowski, Dariusz Zasada, Wojciech Polkowski

The Effect of Aluminum Content on the Microstructure and Cavitation Wear of FeAl Intermetallic Alloys

Tab. 1. FeAl intermetallics alloys subjected to cavitation erosion

\begin{tabular}{|c|c|c|c|c|c|}
\hline \multirow{2}{*}{ Element } & \multicolumn{5}{|c|}{ Intermetallics FeAl alloys } \\
\cline { 2 - 6 } & FeAl36 & FeAl39 & FeAl42 & FeAl45 & FeAl48 \\
\hline $\mathrm{Al}$ & 36.00 & 39.00 & 42.00 & 45.00 & 48.00 \\
\hline $\mathrm{Mo}$ & 0.22 & 0.22 & 0.22 & 0.22 & 0.22 \\
\hline $\mathrm{Zr}$ & 0.10 & 0.10 & 0.10 & 0.10 & 0.10 \\
\hline $\mathrm{B}$ & 0.01 & 0.01 & 0.01 & 0.01 & 0.01 \\
\hline $\mathrm{C}$ & 0.13 & 0.13 & 0.13 & 0.13 & 0.13 \\
\hline $\mathrm{Fe}$ & 63.54 & 60.54 & 57.54 & 54.54 & 51.54 \\
\hline Density $\left[\mathrm{kg} / \mathrm{m}^{3}\right]$ & 6255 & 6068 & 5982 & 5797 & 5687 \\
\hline Hardness HV0.1 & 297.34 & 311.47 & 330.47 & 347.64 & 385.30 \\
\hline
\end{tabular}

\section{METHODS OF INVESTIGATION}

Electron backscattered diffraction system (EBSD) coupled with field emission gun scanning electron microscope (FEG SEM) FEI Quanta 3D was applied to analyze microstructure and microtexture of iron aluminides alloys in as cast state. EBSD analysis was carried out on cross-section of cylindrical ingots (with normal direction (ND) parallel to ingot axis). EBSD data was taken from approx. $1200 \times 1200 \mu \mathrm{m}$ area with step size of $6 \mu \mathrm{m}$ for each investigated sample. A grain boundary character distribution was examined using a misorientation angle in the 2 to $15^{\circ}$ range for low angle grain boundaries (LAGBs) and above $15^{\circ}$ for high angle grain boundaries (HAGBs). Microtexture analysis was performed through computation of orientation distribution functions (ODF) by harmonic expansion method with assumption of axial symmetry of cylindrical sample.

Additionally, EBSD method was applied to estimate strain introduced during cavitational wear of selected alloy. Strain assessment was carried out by local misorientation approach (Kamaya et al., 2005) and represented by Kernel Average Misorientation (KAM) parameter. According to its definition, value of KAM parameter is obtained in following way: for a given data point the average misorientation between the data point and all of its neighbors is calculated (exclude misorientations greater than some prescribed value $-5^{\circ}$ in this case).
The examination of cavitational erosion was carried out on a jet-impact device. Examinated samples had cylindrical shape with $20 \mathrm{~mm}$ diameter and $6 \pm 0.5 \mathrm{~mm}$ height. Surface roughness of samples before experiment, measured by PGM-1C profilometer, was in range of $0.010 \div 0.015 \mu \mathrm{m}$. The samples were vertically mounted in rotor arms, parallel to the axis of water stream pumped continuously at $0.06 \mathrm{MPa}$ through a $10 \mathrm{~mm}$ diameter nozzle located $1.6 \mathrm{~mm}$ away from the sample edge. The rotating samples were hitting by the water stream. Water flow of $1.55 \mathrm{~m}^{3} / \mathrm{h}$ was constant during entire experiment. The samples were examined for the period of 30 minutes, took out from the fixtures, degreased in an ultrasonic washer for 10 minutes at $30^{\circ} \mathrm{C}$, dried in a laboratory drier for 15 minutes at $120^{\circ} \mathrm{C}$ and weighed, than mounted again in the rotor arms, maintaining the initial position in relation to the water stream. The analyses included 2 samples of each alloy, examined for the period of 600 minutes.

\section{RESULTS AND DISCUSSION}
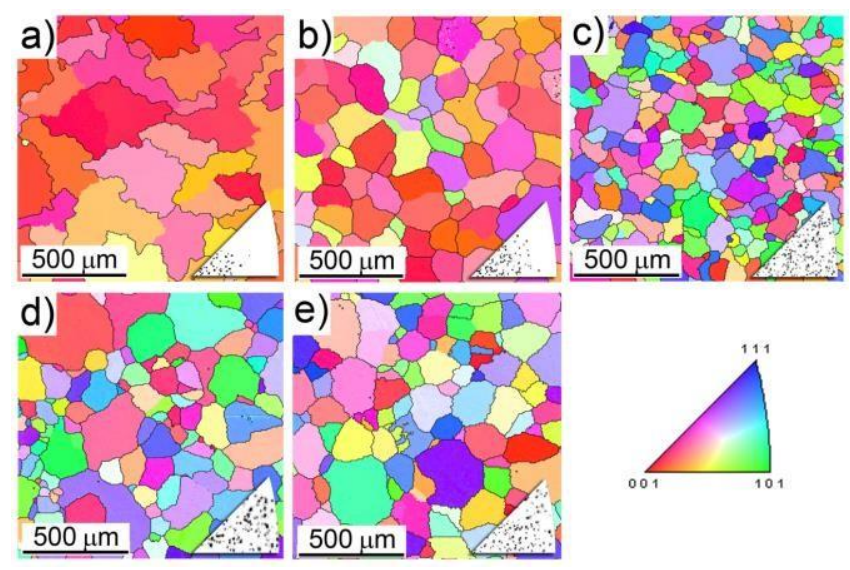

Fig. 1. EBSD inverse pole figure maps taken from a) FeAl36, b) FeAl39, c) FeAl42, d) FeAl45 and e) FeAl48 alloys in as cast state. High angle grain boundaries were marked with thick lines

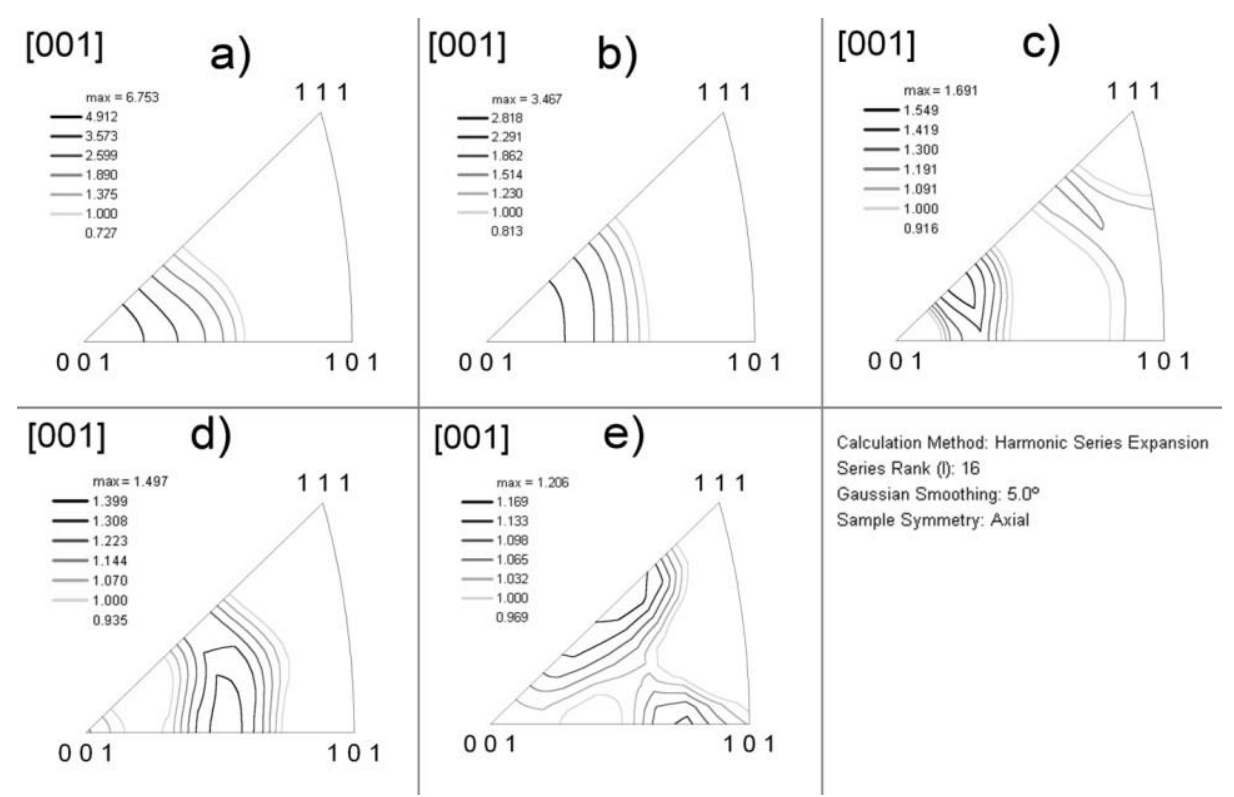

Fig. 2. ODF inverse pole figure calculated for a) FeAl36, b) FeAl39, c) FeAl42, d) FeAl45 and e) FeAl48 alloys in as cast state 
Fig. 1 shows EBSD inverse pole figure maps taken from iron aluminides samples with different aluminum content. According to color code consistent with the based triangle (and discrete inverse pole figures included in each map), it may be found that both FeAl36 and FeAl39 alloys exhibit $<100>/$ /ND axial crystallographic texture (Fig. $3 a$ and $3 b$ ) This kind of texture is typical for most fcc and bcc metals and alloys in as-cast state (Coulomb, 1997). However, obtained results indicate that with increasing aluminum content orientation of grains become more random. The effect of texture weakening is also confirmed by results of ODF calculation presented in Fig. 2. It was shown that maximum ODF values calculated for investigated alloys continuously decreased with rising aluminum content and thus for FeAl42, FeAl45 and FeAl48 (Fig. $2 \mathrm{c}$-e) was almost marginal. This effect may be related with larger aluminium content which results in higher thermal conductivity and the same in better heat dissipation, i.e. in faster crystallisation from liquid phase to solid phase which takes place at a temperature of approximately $1300 \mathrm{oC}$ and the existence of structural defects in the FeAl intermetallic alloys.

FeAl intermetallic alloys samples tested for cavitational erossion resistance carried out on a jet-impact device for 300 minutes were subjected to SEM/EBSD analysis. The effects of plastic straining were observed on the surface of FeAl intermetallic samples (Fig. 3).

a)

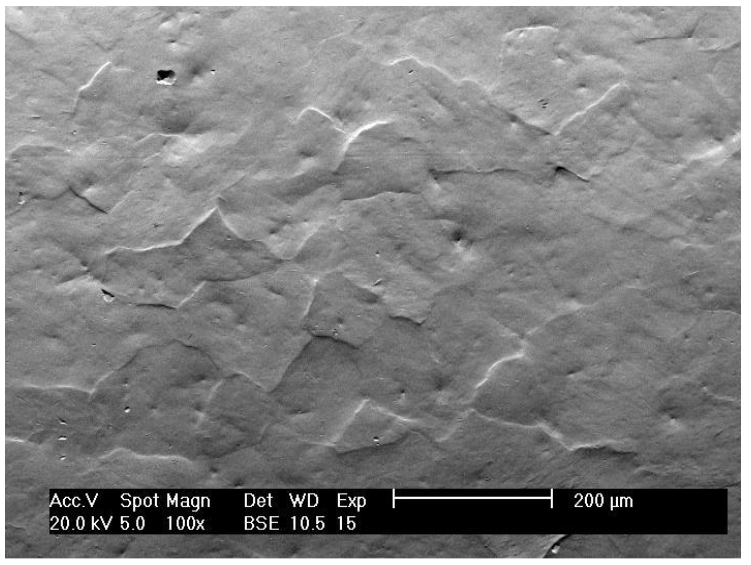

b)

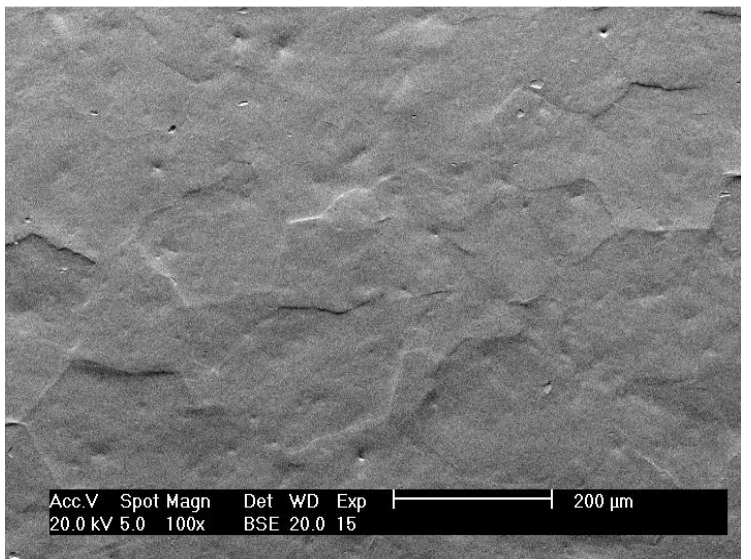

Fig. 3. Effect of plastic strain on the surface a) FeAl39, b) FeAl45

EBSD strain assessment results obtained for Fe-36Al alloy before and after cavitational test are presented in Fig. 4. By comparing inverse pole figure maps presented in Fig. 4a and $4 b$, it may be found that exposition on cavitational beam results with prominent surface area deformation. Effect of cavitational pitting formation was also noted.
Results of KAM mapping of Fe-36Al alloy surface before and after wear test are presented in Fig. $4 \mathrm{c}$ and Fig. 4d, respectively. It is shown that average KAM value calculated for alloy after cavitational examination is significantly raised in comparison with initial material (it was $\sim 7$ times higher). Since this parameter may be directly related with dislocation density (Calcagnatto et al., 2010), this effect indicates that surface of alloy undergoes substantial straining (which is assisted with increase of crystal lattice defects amount). Moreover, results of analysis of KAM values distribution on analyzed surface allows concluding, that strain localization takes place in near grain boundary areas. Obviously, the highest KAM values (marked with red color) were obtained in highly deformed regions that surround observed cavitational pits.
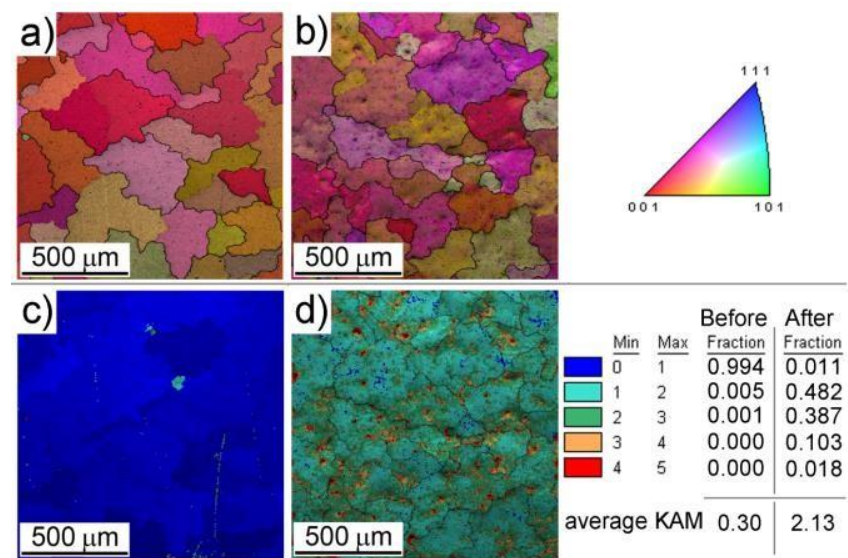

Fig. 4. EBSD inverse pole figure maps taken from a) Fe-36Al alloy surface before cavitational test, b) Fe-36Al alloy surface after cavitational test. c) and d) KAM maps calculated for Fe-36Al surface before and after cavitational test, respectively

Comparison of KAM parameters after cavitational test for FeAl intermatallic alloys samples is presented in Fig. 5.

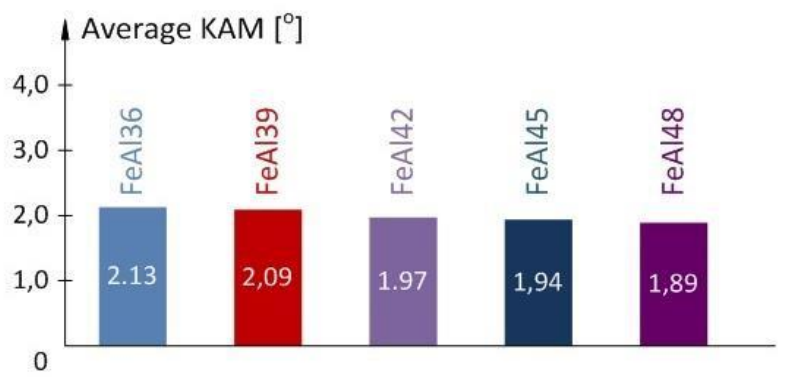

Fig. 5. Comparison of KAM parameter after cavitational test for FeAl intermatallic alloys

The difference in the results of KAM parameter after cavitational wear is dependent on the content of aluminum in FeAl intermetallic alloys. Aluminum content in these alloys will affect the hardness and grain size. Since, mechanical strenght of investigated alloys increases with aluminum content, estimated surface plastic strain decreased with rising fraction of Al. Consequently, Alloys FeAl45 and FeAl48 with greater hardness undergoes to surface plastic strain of lower degree than alloys with smaller content of aluminium. 


\section{CONCLUSION}

Cavitational resistance is defined most frequently by comparison of material destruction kinetics tested on a given installation. The longer is incubation time and the smaller is destruction rate, the larger is the capability of material to resist a destructive effect of cavitation. In the case where the only effect the cavitational destruction is plastic deformation, EBSD strain analysis may be efficiently applied to assess cavitation erosion resistance of material.

Obtained results of EBSD structural characterization indicates that with increasing aluminium content effects of orientation randomization (weakening of $<100>/$ ND casting texture), grain refinement and rising of mechanical strenght (and so cavitational resistance) take place.

\section{REFERENCES}

1. Bojar Z., W. Przetakiewicz W. (2006) Metallic materials with intermetallic phases, Belstudio, (in Polish).

2. Bystrzycki J., Varin R.A., Bojar Z. (1996), The progresses in investigations of intermetallic alloys with aluminum, Inżynieria Materiałowa, Nr 5, 137-148.

3. Blochwitz C., Brechbühl J., Tirschler W. (1996), Analysis of activated slip systems in fatigued nickel polycrystals using the EBSD-technique in the scanning electron microscope, Mater. Sci. Eng. A, 210, 42-47.

4. Calcagnotto M., Ponge D., Demir E., Dierk Raabe D. (2010), Orientation gradients and geometrically necessary dislocations in ultrafine grained dual-phase steels studied by 2D and 3D EBSD, Materials Science and Engineering, A 527, 2738-2746.

5. Coulomb P. (1997), Textures in metals by cubic lattice, Dunod, Paris, (in French).

6. El Bartali A., Aubin V., Sabatier L., Villechaise P., DegallaixMoreuil S. (2008), Identification and analysis of slip systems activated during low-cycle fatigue in a duplex stainless steel, Scr. Mater., 59, 1231-1234.

7. Jasionowski R., Przetakiewicz W., Zasada D. (2011), The effect of structure on the cavitational wear of FeAl intermetallic phasebased alloys with cubic lattice, Archives of Foundry Engineering, Vol. 11, 97-102.

8. Jasionowski R., Przetakiewicz W., Zasada D. (2010), The cavitational erosion resistance of the B2-type Fe-Al casting alloys, Archives of Foundry Engineering, Vol.10, 305-310.

9. Kamaya M., Wilkinson A.J., Titchmarsh J.M. (2005), Measurement of plastic strain of polycrystalline material by electron backscatter diffraction, Nuclear Engineering and Design, 235, 713-725.

10. Schwartz J., Kumar M., Adams L., Field P. (2009), Electron Backscatter Diffraction in Materials Science, second ed., Springer

11. Stella J., Pohl M., Bock C., Kunze U. (2014), Influence of grain orientation on the local deformation mode inducedby cavitation erosion in a CuSnNi alloy, Wear, 316, 1-5.

Acknowledgements: The work has been accomplished under the research project No. N N507 231040 financed by the Scientific Research Committee. 